PROCEEDINGS OF THE AMERICAN MATHEMATICAL SOCIETY

Volume 124, Number 10, October 1996

\title{
MULTIPLE GEOMETRICALLY DISTINCT CLOSED NONCOLLISION ORBITS OF FIXED ENERGY FOR N-BODY TYPE PROBLEMS WITH STRONG FORCE POTENTIALS
}

\author{
ZHANG SHIQING \\ (Communicated by James Glimm)
}

\begin{abstract}
Using the equivariant Ljusternik-Schnirelmann theory, we prove that there are at least $2(N-1) 2^{N-2}$ geometrically distinct noncollision orbits with prescribed energy for a class of planar N-body type problems with strong force potentials.
\end{abstract}

\section{INTRODUCTION}

In the last few years, variational methods have started to be used for studying the periodic solutions for singular Hamiltonian systems. Existence of periodic solutions with prescribed period for some classes of N-body problems has been proved in [2]-[6], [9], [11]. On the contrary, we know of only one paper ([1]) obtaining the results in the large concerning the existence of one periodic solution with prescribed energy, and except for the author's preprint paper ([14]), there is no result in the large dealing with the existence of multiple geometrically distinct closed noncollision orbits. This is mainly because the N-body problems in $\mathbb{R}^{k}$ have $S^{1} \times O(k)$ symmetry, where $O(k)$ is the rotational symmetry group of order $k$. In order to obtain multiple geometrically distinct trajectories, we must consider the effects of the group $S^{1} \times$ $O(k)$; this results in some problems in topology.

Using the equivariant Ljusternik-Schnirelmann theory, we obtain the result in the large concerning multiple geometrically distinct closed noncollision orbits for a class of planar N-body problems with strong force potentials. The key observations of the proof about our results are that the variational functional satisfies the PalaisSmale condition and that we must construct a special set such that its $S^{1} \times O(2)$ equivariant category is easily computed and the superbound of the functional on the set is also easily estimated.

Many variational methods' papers deal with N-body type problems, but assume conditions on the potential that exclude the actual N-body problem (i.e. gravitational potential). This paper is no exception, but in another paper ([14]), the author does deal with gravitational potentials.

Received by the editors January 11, 1995.

1991 Mathematics Subject Classification. Primary 34C25, 34C15, 58F05.

Key words and phrases. N-body type problems with fixed energy, geometrically distinct noncollision periodic orbits, equivariant Ljusternik-Schnirelmann theory.

(c)1996 American Mathematical Society 
We set $\Omega=\mathbb{R}^{2} \backslash\{0\}$ and consider a potential $V$ of the form:

$$
V(x)=V\left(x_{1}, \ldots, x_{N}\right)=\frac{1}{2} \sum_{1 \leq i \neq j \leq N} V_{i j}\left(x_{i}-x_{j}\right),
$$

where $x_{i} \in \mathbb{R}^{2}, x=\left(x_{1}, \ldots, x_{N}\right) \in\left(\mathbb{R}^{2}\right)^{N}$ and $V_{i j} \in C^{1}(\Omega, \mathbb{R})(i, j=1, \ldots, N)$.

Given $m_{i}>0(i=1, \ldots, N)$ and $h \in \mathbb{R}$, we seek periodic solutions of

$$
\begin{cases}m_{i} \ddot{x}_{i}+V_{x_{i}}^{\prime}\left(x_{1}, \ldots, x_{N}\right)=0, & 1 \leq i \leq N, \\ \frac{1}{2} \sum_{i=1}^{N} m_{i}\left|\dot{x}_{i}\right|^{2}+V\left(x_{1}, \ldots, x_{N}\right)=h, & 1 \leq i \leq N .\end{cases}
$$

Definition 1.1. If the solution $x=\left(x_{1}, \ldots, x_{N}\right)$ of $(\mathrm{Ph})$ satisfies

(i) $x_{i} \in C^{2}\left(0, T ; \mathbb{R}^{2}\right)$,

(ii) $x_{i}(t) \neq x_{j}(t), \forall t \in[0, T]$ and $\forall 1 \leq i \neq j \leq N$, then we call $x$ the noncollision periodic solution of $(\mathrm{Ph})$.

Definition 1.2. Let $x$ and $y$ be two periodic solutions of $(\mathrm{Ph})$, neither of which can be brought into the other by the standard $S^{1} \times O(2)$ action. Then we call $x$ and $y$ distinct in geometrics.

Theorem 1.3. Assume $V$ possesses the form of (1.1) and $V_{i j}$ and $V$ satisfy

$(V 1) V_{i j}(\xi)=V_{j i}(\xi), \forall i \neq j, \forall \xi \in \mathbb{R}^{2}$, and $V\left(u_{1}, \ldots, u_{N}\right)=V\left(R u_{1}, \ldots, R u_{N}\right)$, $\forall R \in O(2), \forall\left(u_{1}, \ldots, u_{N}\right) \in\left(\mathbb{R}^{2}\right)^{N}$.

(V2) There are $\alpha>2$ and $a>b>0$ such that for any $x_{i} \in \mathbb{R}^{2}$ and $x_{i} \neq x_{j}$

$$
\begin{gathered}
-\frac{a}{2} \sum_{1 \leq i \neq j \leq N} \frac{m_{i} m_{j}}{\left|x_{i}-x_{j}\right|^{\alpha}} \leq V\left(x_{1}, \ldots, x_{N}\right) \leq-\frac{b}{2} \sum_{i \neq j} \frac{m_{i} m_{j}}{\left|x_{i}-x_{j}\right|^{\alpha}} . \\
c_{2}>\frac{1}{9} c_{5},
\end{gathered}
$$

where

$$
\begin{aligned}
& c_{5}=\frac{1}{2} c_{4}^{2 / \alpha}\left(\frac{\alpha-2}{2}\right)^{2 / \alpha}\left(c_{4}+\frac{2}{\alpha-2} c_{3}\right), \\
& c_{4}=\sum_{i=1}^{N} 4 \pi^{2} i^{2} m_{i}, \\
& c_{3}=\frac{a}{2} \sum_{i \neq j} \frac{m_{i} m_{j}}{|i-j|^{\alpha}}, \\
& c_{2}=\frac{2 \pi^{2} \alpha}{\alpha-2}\left(\frac{\alpha-2}{2}\right)^{2 / \alpha}\left(b c_{1}\right)^{2 / \alpha}, \\
& c_{1}=2^{-(\alpha+2) / 2}\left(\sum_{i \neq j} m_{i} m_{j}\right)^{(\alpha+2) / 2} m^{-\alpha / 2}, \\
& m=\sum_{i=1}^{N} m_{i} .
\end{aligned}
$$

Then for any $h>0,(\mathrm{Ph})$ has at least $2(N-1) 2^{N-2}$ geometrically distinct noncollision periodic solutions. 
Corollary 1.4. In Theorem 1.3 , let $N=3, m_{1}=1, m_{2}=\varepsilon \ll 1, m_{3}=1 / 4, \alpha \rightarrow$ 2 , and $a=b=1$. Then for any $h>0,(\mathrm{Ph})$ has at least 8 geometrically distinct noncollision periodic solutions.

Proof. Under the assumption of Corollary 1.4, we have

$$
\begin{aligned}
& m \approx 5 / 4, \quad c_{1} \approx 1 / 20, \quad c_{2} \approx \pi^{2} / 10, \quad c_{3} \approx 1 / 16, \\
& c_{4} \approx 13 \pi^{2}, \quad c_{5} \approx(1 / 2) c_{3} \times c_{4} \approx(13 / 32) \pi^{2}<9 c_{2} .
\end{aligned}
$$

\section{Functional Setting And the PRoof of Theorem 1.3}

Let us introduce the following notation:

$$
\begin{gathered}
H=W^{1,2}\left(S^{1}, \mathbb{R}^{2}\right), \\
H_{\#}=\left\{u \in H \mid u\left(t+\frac{1}{2}\right)=-u(t)\right\}, \\
E=\left\{u=\left(u_{1}, \ldots, u_{N}\right) \mid u_{i} \in H_{\#}, i=1, \ldots, N\right\}, \\
\Lambda_{0}=\left\{u \in E \mid u_{i}(t) \neq u_{j}(t), \forall t, i \neq j\right\}, \\
\langle u, v\rangle=\int_{0}^{1} \dot{u} \dot{v} d t, \quad\|u\|^{2}=\int_{0}^{1}|\dot{u}|^{2} d t, \quad \forall u, v \in H_{\#} .
\end{gathered}
$$

It is well known that $\left\|u_{i}\right\|$ is a norm which is equivalent to the usual one and we have

$$
\left\|u_{i}\right\| \geq 4\left|u_{i}\right|_{\infty} .
$$

Hence for all $u=\left(u_{1}, \ldots, u_{N}\right) \in E$, setting

$$
\|u\|_{E}^{2}=\sum_{i=1}^{N} m_{i}\left\|u_{i}\right\|^{2},
$$

we obtain

$$
\|u\|_{E}^{2} \geq 16 \underline{m}|u|_{\infty}^{2},
$$

where $\underline{m}=\min \left\{m_{i} \mid i=1, \ldots, N\right\}$ and $|u|_{\infty}^{2}=\sum_{i=1}^{N}\left|u_{i}\right|_{\infty}^{2}$.

On $\Lambda_{0}$ define the following functional:

$$
f(u)=\frac{1}{2}\|u\|_{E}^{2} \int_{0}^{1}(h-V(u)) d t .
$$

Similar to the proof of [1] and [8], we have the following variational principle:

Lemma 2.1. Let $u \in \Lambda_{0}$ be the critical point of $f$ and $\|u\|_{E}>0$. Let

$$
\omega^{2}=\frac{\int_{0}^{1} V^{\prime}(u) \cdot u d t}{\|u\|_{E}^{2}}>0
$$

Then $x(t)=u(\omega t)$ is a noncollision periodic solution of $(\mathrm{Ph})$.

Lemma $2.2([5])$. Let $X=\left(x_{1}, \ldots, x_{N}\right) \in\left(\mathbb{R}^{k}\right)^{N}$. Then

$$
\frac{1}{2} \sum_{1 \leq i \neq j \leq N} \frac{m_{i} m_{j}}{\left|x_{i}-x_{j}\right|^{\alpha}} \geq c_{1} \frac{1}{\left(\sum_{i=1}^{N} m_{i}\left|x_{i}\right|^{2}\right)^{\alpha / 2}} .
$$


Lemma 2.3. Suppose $V$ satisfies $(V 2)$ of Theorem 1.3. Then

$$
-V(x) \geq c \frac{1}{\left(\sum_{i=1}^{N}\left|x_{i}\right|^{2}\right)^{\alpha / 2}} .
$$

Proof. By $\sum_{i=1}^{N} m_{i}\left|x_{i}\right|^{2} \leq \bar{m} \sum_{i=1}^{N}\left|x_{i}\right|^{2}$ and Lemma 2.2, the inequality (2.5) is proved.

Lemma 2.4. Assume $(V 1)$ and $(V 2)$ hold. Then $f \in C^{1}\left(\Lambda_{0}, \mathbb{R}\right)$ and

$$
f\left(u_{n}\right) \rightarrow+\infty, \quad \forall u_{n} \rightarrow u \in \partial \Lambda .
$$

Proof. (i) If $u(t) \equiv 0$, then by the embedding theorem,

$$
\left|u_{n}\right|_{\infty} \rightarrow 0, \quad n \rightarrow \infty
$$

Hence by inequality (2.1), $(V 2)$ and Lemma 2.3 , we have

$$
\begin{aligned}
f\left(u_{n}\right) & =\frac{1}{2}\left\|u_{n}\right\|_{E}^{2} \int_{0}^{1}\left(h-V\left(u_{n}\right)\right) d t \geq \frac{1}{2}\left\|u_{n}\right\|_{E}^{2} \int_{0}^{1}\left(-V\left(u_{n}\right)\right) d t \\
& \geq 8 \underline{m}\left|u_{n}\right|_{\infty}^{2} c \int_{0}^{1}\left|u_{n}\right|^{-\alpha} d t \geq 8 \underline{m} c\left|u_{n}\right|_{\infty}^{2}\left|u_{n}\right|_{\infty}^{-\alpha} .
\end{aligned}
$$

Hence $f\left(u_{n}\right) \rightarrow+\infty$ as $n \rightarrow \infty$.

(ii) If $u(t) \not \equiv 0$, then $\|u\|_{E}^{2}=\sum_{i=1}^{N} m_{i}\left\|\dot{u}_{i}\right\|_{2}^{2} \neq 0$. In fact, if $\|u\|_{E}=0$, then $u_{i}(t) \equiv c=0$ by $u_{i}\left(t+\frac{1}{2}\right)=-u_{i}(t)$. By the weak lower semi-continuous property of $g(u)=\|u\|_{E}$, we have

$$
\liminf _{n \rightarrow \infty}\left\|u_{n}\right\|_{E} \geq\|u\|_{E}>0 .
$$

By $(V 2), V_{i j}$ satisfies the strong force condition of Gordon ([7]), and so

$$
\int_{0}^{1} V\left(u_{n}\right) d t \rightarrow-\infty, \quad \forall u_{n} \rightarrow u \in \partial \Lambda_{0} .
$$

Hence by (2.8) and (2.9), we have

$$
f\left(u_{n}\right)=\frac{1}{2}\|u\|_{E}^{2} \int_{0}^{1}\left(h-V\left(u_{n}\right)\right) d t \rightarrow+\infty, \quad n \rightarrow \infty .
$$

Lemma 2.5. $f$ satisfies the Palais-Smale condition in $\Lambda_{0}$.

Proof. Assume $\left\{u_{n}\right\} \subset \Lambda_{0}$ and that it satisfies

$$
\begin{gathered}
\left|f\left(u_{n}\right)\right| \leq M, \\
f^{\prime}\left(u_{n}\right) \rightarrow 0 .
\end{gathered}
$$

Then by $(2.10)$ and $V\left(u_{n}\right) \leq 0$, we know

$$
0 \leq-\frac{1}{2}\left\|u_{n}\right\|_{E}^{2} \int_{0}^{1} V\left(u_{n}\right) d t \leq M-\frac{h}{2}\left\|u_{n}\right\|_{E}^{2} ;
$$

hence $\left\|u_{n}\right\|$ is bounded, and there is a weakly convergent subsequence $\left\{u_{n_{k}}\right\}$ of $\left\{u_{n}\right\}$. Similar to the proof of [1], $u_{n_{k}} \rightarrow u \in \Lambda_{0}$, and hence $f$ satisfies the Palais-Smale condition.

Lemma 2.6. On $\Lambda_{0}, f$ possesses a positive lower bound:

$$
\inf _{u \in \Lambda_{0}} f(u) \geq c_{2} .
$$


Proof. By Lemma 2.2, for any $u \in \Lambda_{0}$, we have that

$$
f(u) \geq \frac{1}{2} \int_{0}^{1}\left(\sum_{i=1}^{N} m_{i}\left|\dot{u}_{i}\right|^{2}\right) d t\left(h+b c_{1} \int_{0}^{1}\left(\sum_{i=1}^{N} m_{i}\left|u_{i}\right|^{2}\right)^{-\alpha / 2} d t\right) .
$$

By the Hölder inequality, we have

$$
\int_{0}^{T}|q| d t \leq T^{1 / 2}\left(\int_{0}^{T}|q|^{2} d t\right)^{1 / 2}, \quad\left(\int_{0}^{T}|q|^{2} d t\right)^{\alpha / 2} \geq T^{-\alpha / 2}\left(\int_{0}^{T}|q| d t\right)^{\alpha} .
$$

By the Chebychev inequality, we have

SO

$$
\int_{0}^{T} \frac{1}{|q|} d t \cdot \int_{0}^{T}|q| d t \geq T^{2}
$$

$$
\left(\int_{0}^{T} \frac{1}{|q|} d t\right)^{\alpha} \geq T^{3 \alpha / 2}\left(\int_{0}^{T}|q|^{2} d t\right)^{-\alpha / 2}
$$

Using the above results and the Jensen inequality, we have

$$
\begin{gathered}
\int_{0}^{T} \frac{1}{|q|^{\alpha}} d t \geq T\left(\frac{1}{T} \int_{0}^{T} \frac{1}{|q|} d t\right)^{\alpha} \geq T^{1+\alpha / 2}\left(\int_{0}^{T}|q|^{2} d t\right)^{-\alpha / 2} \\
\int_{0}^{T}\left(\sum_{i=1}^{N} m_{i}\left|u_{i}\right|^{2}\right)^{-\alpha / 2} d t \geq T^{1+\alpha / 2}\left[\int_{0}^{T}\left(\sum_{i=1}^{N} m_{i}\left|u_{i}\right|^{2}\right) d t\right]^{-\alpha / 2}
\end{gathered}
$$

By the Wirtinger inequality, we have that

$$
f(u) \geq 2 \pi^{2} \int_{0}^{1}\left(\sum_{i=1}^{N} m_{i}\left|u_{i}\right|^{2}\right) d t\left\{h+b c_{1}\left[\int_{0}^{1}\left(\sum_{i=1}^{N} m_{i}\left|u_{i}\right|^{2}\right) d t\right]^{-\alpha / 2}\right\} .
$$

Let $r^{2}=\int_{0}^{1}\left(\sum_{i=1}^{N} m_{i}\left|u_{i}\right|^{2}\right) d t$ and $g(r)=2 \pi^{2} r^{2}\left(h+b c_{1} r^{-\alpha}\right)$. Then $f(u) \geq$ $\min _{r>0} g(r)$ since $r=r_{0}=\left[(\alpha-2) b c_{1} / 2 h\right]^{1 / \alpha}$ satisfies $g^{\prime}\left(r_{0}\right)=0$ and $g^{\prime \prime}\left(r_{0}\right)>0$. Hence $g(r)$ has minimum value $g\left(r_{0}\right)$ and

$$
f(u) \geq g\left(r_{0}\right)=\frac{2 \pi^{2} \alpha}{\alpha-2}\left(\frac{\alpha-2}{2}\right)^{2 / \alpha}\left(b c_{1}\right)^{2 / \alpha} \cdot h^{(\alpha-2) / \alpha} \equiv c_{2} h^{(\alpha-2) / \alpha} .
$$

In the following, we define a $S^{1} \times O(2)$ invariant set $A$ and estimate the above bound of $f$ on $A$. Let

$$
\begin{aligned}
Z_{i} & =\left\{\nu(t)=\operatorname{Ri}(\xi \cos 2 \pi t+\eta \sin 2 \pi t)\left|\xi, \eta \in \mathbb{R}^{2},\right| \xi|=| \eta \mid=1,\langle\xi, \eta\rangle=0\right\} \\
A & =Z_{1} \times \cdots \times Z_{N} .
\end{aligned}
$$

Then for any $u=\left(u_{1}, \ldots, u_{N}\right) \in A$, there are $\xi_{i}, \eta_{i} \in \mathbb{R}^{2}$ such that $\left|\xi_{i}\right|=\left|\eta_{i}\right|=1$, $\xi_{i} \cdot \eta_{i}=0$ and $u_{i}(t)=R i\left(\xi_{i} \cos 2 \pi t+\eta_{i} \sin 2 \pi t\right)$. Hence $\left|u_{i}(t)\right|^{2}=R^{2} i^{2}$ and

$$
\begin{aligned}
\left|u_{i}(t)-u_{j}(t)\right|^{2} & \geq\left|u_{i}(t)\right|^{2}+\left|u_{j}(t)\right|^{2}-2\left|u_{i}(t)\right| \cdot\left|u_{j}(t)\right| \\
& =R^{2}\left(i^{2}+j^{2}-2 i j\right)=[R(i-j)]^{2} .
\end{aligned}
$$


Hence by $(V 2)$, we have

$$
-V(u) \leq \frac{1}{R^{\alpha}}\left(\frac{a}{2} \sum_{i \neq j} \frac{m_{i} m_{j}}{|i-j|^{\alpha}}\right)=c_{3} \cdot \frac{1}{R^{\alpha}} .
$$

It is easily seen that

$$
\begin{gathered}
\dot{u}_{i}=2 \pi R i\left(-\xi_{i} \sin 2 \pi t+\eta_{i} \cos 2 \pi t\right) \\
\|u\|_{E}^{2}=\sum_{i=1}^{N} m_{i} \int_{0}^{1}\left|\dot{u}_{i}\right|^{2} d t=R^{2}\left(\sum_{i=1}^{N} 4 \pi^{2} i^{2} m_{i}\right)=c_{4} R^{2} .
\end{gathered}
$$

By the above estimates, we have

Lemma 2.7. If we choose the $R$ of the set $A$ as $R^{*}=\left[(\alpha-2) c_{4} / 2 h\right]^{1 / \alpha}$, then

$$
\max _{u \in A} f(u) \leq c_{5} .
$$

Proof.

$$
\begin{aligned}
f(u) & =\frac{1}{2}\|u\|_{E}^{2} \cdot \int_{0}^{1}(h-V(u)) d t \leq \frac{1}{2} c_{4} R^{2} \int_{0}^{1}\left(h+c_{3} \frac{1}{R^{\alpha}}\right) d t \\
& =\frac{1}{2} c_{4} h R^{2}+\frac{1}{2} c_{3} c_{4} R^{2-\alpha}=g(R) .
\end{aligned}
$$

Then $R=R^{*}=\left[(\alpha-2) c_{4} / 2 h\right]^{1 / \alpha}$ satisfies $g^{\prime}\left(R^{*}\right)=0, g^{\prime \prime}\left(R^{*}\right)>0$. So $g(R)$ has unique minimum value

$$
g\left(R^{*}\right)=\frac{1}{2} c_{4}^{2 / \alpha}\left(\frac{\alpha-2}{2}\right)^{2 / \alpha} h^{(\alpha-2) / \alpha}\left(c_{4}+\frac{2}{\alpha-2} c_{3}\right) \equiv c_{5} h^{(\alpha-2) / \alpha} .
$$

In the following, we apply the equivariant Ljusternik-Schnirelmann theory to prove the existence of multiple geometrically distinct periodic solutions for $(\mathrm{Ph})$.

We note that $A$ is $S^{1} \times O(2)$ invariant, in fact,

$$
H(\tau, R) \cdot x(t) \equiv\left(R x_{1}(t+\tau), \ldots, R x_{N}(t+\tau)\right) \in A
$$

for any $x(t)=\left(x_{1}(t), \ldots, x_{N}(t)\right), \tau \in S^{1}$ and $R \in O(2)$. We also note that the functional $f$ is $S^{1} \times O(2)$ invariant. Hence in order to obtain multiple distinct $S^{1} \times O(2)$ invariant orbits of the system $(\mathrm{Ph})$, we need to estimate the $S^{1} \times O(2)$ equivariant category of the special set $A$. We note that each $Z_{i}(i=1, \ldots, N)$ is diffeomorphic to $T_{1} S^{1}$, which is the unit tangent bundle of $S^{1}$. Hence cat $\left(Z_{i}\right) \geq 3$. Now $A=Z_{1} \times \cdots \times Z_{N}$ is diffeomorphic to $T_{1} S^{1} \otimes \cdots \otimes T_{1} S^{1}$ ( $N$ factors), and $A$ is diffeomorphic to the set $Z_{\left(n_{1}, \ldots, n_{N}\right)}$ of Coti Zelati ([4]), so by [4], we have

Lemma 2.8. $\operatorname{cat}\left(A / S^{1} \times O(2)\right) \geq 2(N-1) 2^{N-2}$.

Remark. Although the set $A$ is diffeomorphic to the critical manifold $Z_{\left(n_{1}, \ldots, n_{N}\right)}$ of Coti Zelati ([4]), the above bound of $f$ on $A$ is more easily estimated than on $Z_{\left(n_{1}, \ldots, n_{N}\right)}$.

Lemma 2.9. Let $X$ be a Banach space, $\Lambda$ an open subset of $X$ and $f \in C^{1}(\Lambda, R)$. Let $G$ be a compact Lie group, $T(G)$ a linear continuous representation with equivariant distance and $M a C^{2-0}$ submanifold of $\Lambda$. Assume $M$ and $f$ both are 
invariant under $T(G), f$ satisfies the Palais-Smale condition on some closed subset $N$ of $M$ and the following boundary condition holds:

$$
f\left(u_{n}\right) \rightarrow+\infty, \quad \forall u_{n} \rightarrow u \in \partial \Lambda .
$$

Let $i$ be a $T(G)$-invariant index. Let $c_{m}=\inf _{i(A) \geq m} \sup _{x \in A} f(x), m=1,2, \ldots$, where $A \subset N, A \in \Sigma=\{B \subset N \mid B$ is $T(G)$-invariant and closed in $N\}$. Then:

(1) When $-\infty<c_{m}<+\infty, c_{m}$ is a critical value of $f$.

(2) If $-\infty<c=c_{m+1}=\cdots=c_{m+k}<+\infty$, then $i\left(K_{c}\right) \geq k$, where $K_{c}=\{x \in$ $\left.N \mid f^{\prime}(x)=0, f(x)=c\right\}$.

(3) $c_{m} \leq c_{m+1}$.

We note that, from the boundary condition (B.C.) and the Palais-Smale condition, one can deduce the complete property of the open set $\Lambda$, that is, for any $\left\{x_{n}\right\} \subset \Lambda$ such that $x_{n} \in f_{c}=\{x \in \Lambda \mid f(x) \leq c\}$ and $x_{n} \rightarrow x$, we have $x \in \Lambda$. The rest of the proof is standard (see Rabinowitz [10]).

Lemma 2.10. If $u$ is a critical point of $f$ in $\Lambda_{0}$ which has minimal period $\frac{1}{l}, l \neq 1$, then

(i) $l \geq 3$

(ii) $v(t)=u(t / l)$ is also a critical point of $f$ in $\Lambda_{0}$ and $f(v)=\frac{1}{l^{2}} f(u)<\frac{1}{9} f(u)$.

Proof. (i) If $l=2$, then $u\left(t+\frac{1}{2}\right)=u(t)$. By $u \in \Lambda_{0}$, we have $u\left(t+\frac{1}{2}\right)=-u(t)$. Hence $u(t)=\left(u_{1}(t), \ldots, u_{N}(t)\right) \equiv 0$; this contradicts $u \in \Lambda_{0}$.

(ii)

$$
\begin{gathered}
\int_{0}^{1}|\dot{v}(t)|^{2} d t=\frac{1}{l^{2}} \int|\dot{u}(t)|^{2} d t, \\
\int_{0}^{1}(h-V(v)) d t=\int_{0}^{1}(h-V(u)) d t, \\
f(v)=\frac{1}{l^{2}} f(u) \leq \frac{1}{9} f(u) .
\end{gathered}
$$

Now Theorem 1.3 can be proved by Lemma 2.1 and Lemmas $2.4-2.10$.

\section{ACKNOWLEDGEMENT}

The author wishes to thank the referee for useful suggestions.

\section{REFERENCES}

[1] A. Ambrosetti and V. Coti Zelati, Closed orbits of fixed energy for a class of $N$-body problems, Ann. IHP Analyse Nonlineaire 9 (1992), 187-200. MR 93e:58156a

[2] A. Bari and P. H. Rabinowitz, Periodic solutions of Hamiltonian systems of 3-body type, Ann. IHP Analyse Nonlineaire 8 (1991), 561-649. MR 92k:58223

[3] U. Bessi and V. Coti Zelati, Symmetries and non-collision closed orbits for planar $\mathrm{N}$-body type problems, J. Nonlinear Anal. T.M.A. 16 (1991), 587-598. MR 92a:70006

[4] V. Coti Zelati, A class of periodic solutions of the N-body problem, Celestial Mech. 46 (1989), 177-186. MR 91c:58116

[5] — The periodic solutions of N-body type problems, Ann. IHP Analyse Nonlineaire 7 (1990), 477-492. MR 93a:70009

[6] E. Fadell and S. Husseini, Infinite cuplength in free loop spaces with an application to a problem of the N-body type, Ann. IHP Analyse Nonlineaire 9 (1992), 305-319. MR 94a:58034

[7] W. Gordon, Conservative dynamical systems involving strong forces, Trans. Amer. Math. Soc. 204 (1975), 113-135. MR 51:14152 
[8] E. W. C. Van Groesen, Analytical min-max methods for Hamiltonian break orbits of prescribed energy, J. Math. Anal. Appl. 132 (1988), 1-12. MR 89m:58062

[9] P. Majer and S. Terracini, Periodic solutions to some N-body type problems, preprint, 1992.

[10] P. Rabinowitz, Minimax methods in critical point theory with applications to differential equations, C.B.M.S. Reg. Conf. Ser. in Math. 65 (1986). MR 87j:58024

[11] E. Serra and S. Terracini, Noncollision solutions to some three-body problems, Arch. Rational Mech. Anal. 120 (1992), 305-325. MR 93i:70013

[12] _ Noncollision solutions to some singular minimization problems with Keplerian-like potentials, Nonlinear Analysis T.M.A. 22 (1994), 45-62. MR 94m:58091

[13] C. L. Siegel and K. Moser, Lectures on celestial mechanics, Springer-Verlag, 1971. MR 58:19464

[14] S. Q. Zhang, Multiple closed orbits of fixed energy for $N$-body-type problems with gravitational potentials, preprint.

Department of Applied Mathematics, Chongqing University, Chongqing 630044, PeoPLE'S REPUBLIC OF CHINA

E-mail address: cul@cbistic.sti.ac.cn 